\title{
Magnetoresistance in a grid-type lateral superlattice: the role of disorder
}

\author{
D. Weiss ${ }^{a, b}$, A. Menschig ${ }^{c}, K$. von Klitzing ' and G. Weimann " \\ "Bellcore, Red Bank, NJ 07701, USA \\ " Max-Planck-Institut für Festkörperforschung, Stuttgart, Germany \\ "IV Physikalisches Institut der Unicersität Stuttgart, Stuttgart, Germany \\ "Walter-Schottky-Institut der TU München. München. Germany.
}

Received 21 May 1991; accepted for publication 26 August 1991

\begin{abstract}
The magnetoresistance of a two-dimensional electron gas (2DEG) subjected to a weak two-dimensional (2D) lateral superlattice potential is expected to reflect the peculiar self-similar energy spectrum of Bloch electrons in at magnetic field, i.c.. the splitting of Landau levels (LL's) into sublevels. One experimentally established effect of a periodic potential is that the discrete LL's are transformed into Landau bands and cause magnetoresistance oscillations due to an extra band-conductivity contribution. Theoretically, this band-conductivity contribution is expected to be suppressed if the splitting of the Landau bands is resolved. We study the suppression of the band-conductivity as a function of the electron mobility at millikelvin temperatures. Microfabricated gates, defined on top of high mobility GaAs / AIGaAs heterojunctions, are used to electrostatically impose a 2D-periodic potential with $150 \mathrm{~nm}$ period upon the 2DEG. By applying a positive bias the electron mobility is increased and we find the band-conductivity effectively suppressed if the mobility is well above $1 \times 10^{6} \mathrm{~cm}^{2} / \mathrm{Vs}$.
\end{abstract}

In the presence of a weak one-dimensional (1D) lateral superlattice potential the magnetoresistance of a $2 D E G$ displays two types of oscillations reflecting the commensurability between cyclotron radius $R_{\mathrm{c}}$ and period $a$ [1]. Measuring the magnetoresistance parallel ( $y y$-component) and perpendicular ( $x x$-component) to the 1D-modulation, both types are clearly distinguishable. Minima in $\rho_{x}$ are always observed when

$2 R_{\mathrm{c}}=(\lambda-1 / 4) a \quad \lambda=0,1,2, \ldots$,

while, for that condition, maxima are observed in $\rho_{y,}$. Both types originate from transforming the highly degenerate Landau levels into Landau bands with a dispersion dependent on the center coordinate $x_{0}$. This bandwidth $U_{n}\left(x_{0}\right)$ oscillates with respect to the quantum number $n$ and the magnetic field $B$ (sec e.g., ref. [2]). Within first order perturbation theory, eq. (1) describes the magnetic field values where the bands become flat $\left(U_{n}=0\right)$. The oscillatory behaviour of $\rho_{y y}$ simply reflects the modification of the density of states (DOS), and maxima in $\rho_{y y}$ occur when the bands are flat, corresponding to a maximum in the DOS [3]. According to Gerhardts [3a], this effect is due to an oscillating scattering rate, the same mechanism which leads to Shubnikov-de Haas $(\mathrm{SdH})$ oscillations in an unmodulated 2DEG [4]. This mechanism is isotropic, i.c., does not depend on the direction of current flow. The $\rho_{x x}$ component, however, is dominated by the oscillatory behaviour of an additional band-conductivity mechanism [2,5]. For a modulation in $x$-direction. the $x_{10}$-dispersion of the Landau bands opens up an extra conductivity channel: a non-vanishing group velocity $r_{1} \propto \mathrm{d} E_{n} / \mathrm{d} x_{0}$ causes enhaneed conductivity in $y$-direction but not in $x$-direction $\left(v_{x}=0\right)$. Note that for $\omega_{c} \gg 1, \sigma_{x, x} \propto \rho_{y y}$ and $\sigma_{1}$ $\propto \rho_{x x}$. Since $\mathrm{d} E_{n} / \mathrm{d} x_{11}=0$ for flat bands, $\rho_{x,}$ displays minima when eq. (1) holds. The bandconductivity mechanism can also be derived in terms of a semi-classical picture not taking into account LL quantization [6]. The electric ficlds connected to a periodic modulation in $x$-direc- 
tion cause an $\boldsymbol{E} \times \boldsymbol{B}$ drift in $y$-direction. The square of the drift velocity, averaged over all possible center coordinates, is finite and does vanish only when eq. (1) is valid. Within a Boltzmann transport picture the oscillatory behaviour of $\rho_{x x}$ (but not of $\rho_{y y}$ ) can be described semiclassically. Extending this picture to the case of a weak 2D-periodic potential gives the same answer: an additional cosine potential in $y$-direction only results in an additional drift-velocity contribution in $x$-direction but both velocities become zero when $2 R_{c}=(\lambda-1 / 4) a$. From this picture we expect band-conductivity oscillations also in the 2D-modulated case. The situation is more complex if the problem is treated quantum-mechanically. As in the semi-classical case a modulation $V_{0}(\cos (K x) \mid \cos (K y))$ with the same $K=$ $2 \pi / a$ in both lateral directions is considered. Two commensurability effects are effective. In the first place, the Landau bandwidth oscillates in the same mathematical manner as in 1D-modulated 2DEG's. For high Landau quantum numbers $n$, the bandwidth $U_{n}$ is essentially proportional to $V_{0} \pi^{-1 / 2}(n X)^{-1 / 4} \cos (2 \sqrt{n X}-\pi / 4)[2,5]$. Here $V_{0}$ is the modulation amplitude, assumed small compared to $\hbar \omega_{c}$, and $X=K^{2} l^{2} / 2$ with $l=(\hbar / \mathrm{e} B)^{1 / 2}$, the magnetic length. Secondly, the additional modulation in $y$-direction couples Landau states with center coordinates differing by integer multiples of $l^{2} K$ and each Landau band splits into $p$ subbands if

$B a^{2} / \Phi_{0} \equiv a^{2} / 2 \pi l^{2}=p / q$,

i.e., if the flux $\Phi=B a^{2}$ per unit cell is a rational multiple of the flux quantum $\Phi_{0}=h / \mathrm{e}[7,8]$. It is this Landau level subband splitting that can causes the destruction of the band-conductivity contribution if the intrinsic collision broadening, due to random scattering events, is small enough [9]. If the subbands become resolved with respect to impurity broadening (thermal smearing does not matter), the velocity matrix elements ("group velocity") between the subbands do not contribute and the band-conductivity contribution is suppressed. If, on the other hand, collision broadening prevents the resolution of the LL-subbands the band-conductivity contribution is effective again $[9,11]$. The suppression of the band-conduc- a

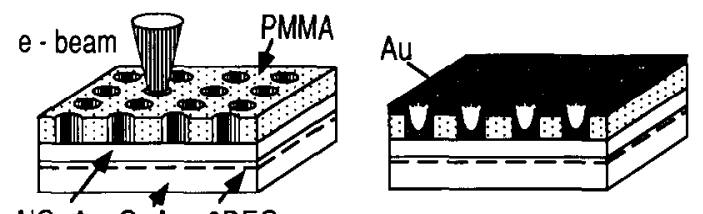

AlGaAs GaAs 2DEG

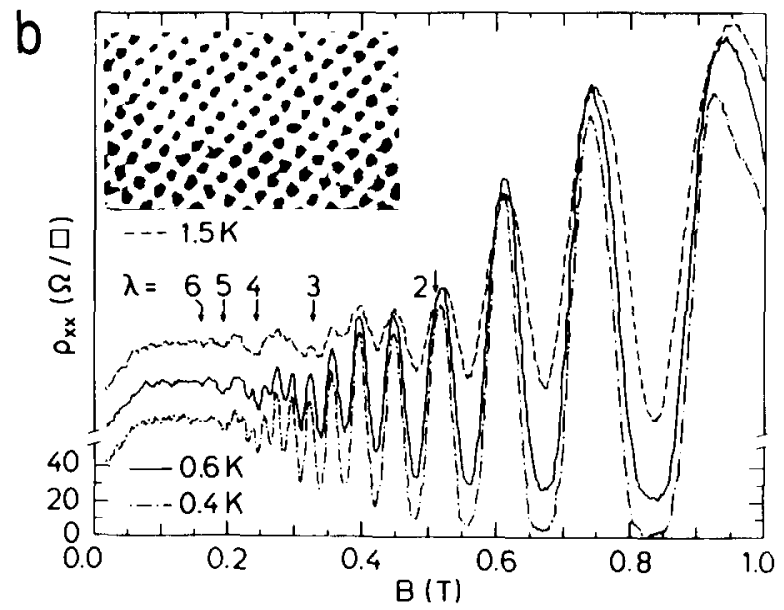

Fig. 1. Sketch of the micropattern fabrication using e-beam lithography (a). An electron micrograph of the micropatterned gate is shown in the inset of (b). The data are taken for $V_{\mathrm{g}}=-200 \mathrm{mV}$ applied to the gate with a $150 \mathrm{~nm}$ stitch. When the temperature is lowered from 1.5 to $0.4 \mathrm{~K}$, amplitude-modulated SdH-oscillations emerge from the band-conductivity oscillations and demonstrate their quantum-mechanical origin. The traces are shifted vertically. Arrows indicate flat bands; magnetoresistance minima at flatband positions are characteristic for band-conductivity oscillations. The mobility $\mu=950000 \mathrm{~cm}^{2} / \mathrm{Vs}$ is not high enough to suppress the dominating band-conductivity. Here $N_{s}=1.6 \times 10^{11} \mathrm{~cm}^{-2}$.

tivity in high-mobility samples has been demonstrated recently by using the in situ-holographic illumination technique $[10,9]$. In this contribution we investigate experimentally the evolution from a dominating band-conductivity to an oscillating scattering rate controlled conductivity mechanism by changing the electron mobility.

Magnetoresistance is measurcd in a $2 \mathrm{D}$-pcriodic potential with lattice constant $a=150 \mathrm{~nm}$. For this periodicity $B a^{2}$, the flux through the unit cell, is increased by one flux quantum, $h / \mathrm{e}$, if the magnetic field is increased by $\sim 0.18 \mathrm{~T}$. Electron beam lithography has been used to define the lateral superlattice on high mobility GaAs / $\mathrm{AlGaAs}$ heterojunctions as is skctched in fig. 1a. In a first step a periodic array of dots is written 
by the focused electron beam. After developing, an array of holes remains in the PMMA. The whole patterned area is covered with an evaporated Au-layer, used as a top gate. An electron micrograph of the micropatterned gate is shown in the inset of fig. 1b. An applied positive or negative bias results in a periodic modulation of the 2DEG underneath. Even without biasing a modulation is present: the metal-semiconductor contact at the bottom of each hole changes the band bending due to differences in the work functions and results in a built-in periodic potential. A slight reduction of the carrier density in samples with Schottky gates is usually observed. Data from two samples are shown here. Both devices have a carrier density $N_{\mathrm{s}} \sim 2 \times 10^{11} \mathrm{~cm}^{-2}$ and a mobility $\mu$ around $1.5 \times 10^{6} \mathrm{~cm}^{2} / \mathrm{Vs}$ in the ungated areas. Both have a $2.4 \mu \mathrm{m}$ GaAs buffer layer and a $20 \mathrm{~nm}$ GaAs cap layer; sample A (B) has a $34 \mathrm{~nm}(28 \mathrm{~nm})$ thick $\mathrm{AlGaAs}$ spacer, and a $34 \mathrm{~nm}(40 \mathrm{~nm})$ Si-doped $\left(\sim 5 \times 10^{18} \mathrm{~cm}^{-3}\right) \mathrm{Al}-$ GaAs layer. The total distance between surface and 2DEG for both samples therefore was $88 \mathrm{~nm}$.

For an applied negative gate bias of $-200 \mathrm{mV}$, $\rho_{x x}$ is displayed for three different temperatures in fig. $1 \mathrm{~b}$. At $1.6 \mathrm{~K}$, band-conductivity oscillations are observed at low fields where $\mathrm{SdH}$-oscillations are still not resolved due to thermal broadening of the LL's $\left(k T \geq \hbar \omega_{c}\right)$. The arrows mark the flatband condition (eq. (1)): the cyclotron radius at the Fermi energy $E_{\mathrm{F}}, R_{\mathrm{c}}=\hbar \sqrt{2 \pi N_{\mathrm{s}}} / \mathrm{e} B$, has been calculated from $N_{\mathrm{s}}$, deduced from the SdH-oscillations at higher fields. At magnetic fields below $0.3 \mathrm{~T}$ the $1.5 \mathrm{~K}$-data meet the semiclassical expectation [11]: Landau quantization is not resolved and minima in $\rho_{x x} \equiv \rho_{y y}$ are observed when eq. (1) holds. If the temperature is lowered, however, SdH-oscillations emerge at lower $B$ values and it becomes obvious that the periodic potential modulates the amplitude of the SdH-oscillations. Minima in the envelope of the oscillations at flatband condition indicate the dominating band-conductivity contribution.

The band-conductivity contribution now can be destroyed if a positive gate voltage is applied. One effect of the positive bias is that the carrier density and the mobility is increased. In the simplest approximations, the mobility $\mu$ is directly

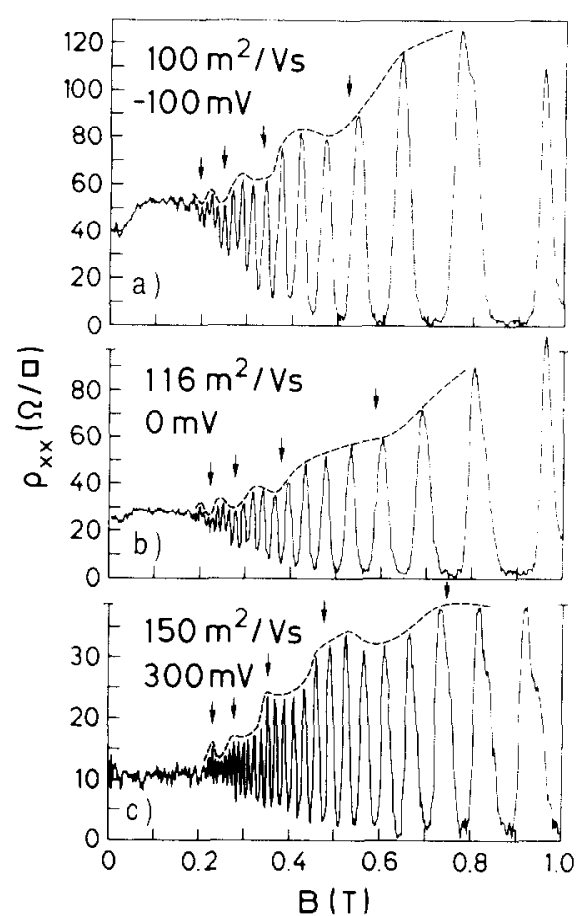

Fig. 2. Suppression of the band-conductivity contribution (maxima between flat bands) when the mobility is raised from $1 \times 10^{6} \mathrm{~cm}^{2} / \mathrm{Vs}$ (a) to $1.5 \times 10^{6} \mathrm{~cm}^{2} / \mathrm{Vs}$ (c). Maxima in the amplitude of the $\mathrm{SdH}$-oscillations remain at flat bands, marked by arrows. The data are taken at $-50 \mathrm{mK}$ using standard lock-in techniques $\left(f \sim 13 \mathrm{~Hz}, I_{\text {meas }}=10 \mathrm{nA}\right.$, 4-point measurements).

connected to a collision broadened linewidth $\Gamma[\mathrm{meV}]=1.37 \times \sqrt{B[\mathrm{~T}] / \mu\left[\mathrm{m}^{2} / \mathrm{Vs}\right]}$ [4]. An increased $\mu$ therefore reduces the intrinsic linewidth $\Gamma$ (caused by random scattering events) and the internal LL-structure (subband splitting) becomes better resolved. We expect an applied bias also to influence the built in periodic potential. The gaps between LL-subbands are proportional to the bandwidth $U_{n}$ and $V_{0}$ directly determines the gap size. $V_{0}$, however, is not a very well-known parameter which is also due to the fact that the "built-in" periodic potential (at zero gate voltage) is not known. Applying a positive bias may reduce the periodic potential since the electron density underneath the Schottky-barriers is slightly reduced.

The sequence of data displayed in fig. 2 is taken at a temperature of $\sim 50 \mathrm{mK}$. For gate 
voltages $V_{\mathrm{g}}=-100 \mathrm{mV}$ and $V_{\mathrm{g}}=0 \mathrm{mV}$ the band-conductivity dominates: minima in the envelope of the $\mathrm{SdH}$-oscillations for flat bands are clearly displayed. For $V_{\mathrm{g}}=+300 \mathrm{mV}$ the phase of the oscillations has changed and the highest maxima of the $\mathrm{SdH}$-oscillations coincide with flat bands. This indicates the suppression of the band-conductivity (also found in holographically illuminated high mobility samples $[10,9]$ ) and the maxima now simply reflect the highest DOS maxima. The switching from one to the other mechanism occurs around $+100 \mathrm{mV}$. Note that the minima of the SdH-oscillations which are a measure of the DOS between LL's are especially deep around the marked flat band conditions in all traces. This demonstrates that flat bands are still correctly described by eq. (1) even for an applied bias of $+300 \mathrm{mV}$. We interprete the suppression of the band-conductivity as consequence of the LL splitting in a weak 2D-periodic potential: the increase of the mobility and the change of the built in potential creates a condition in which the subband splitting becomes resolved and therefore effective. The switching from band-conductivity to the oscillating scattering rate contribution can be verified theoretically. Calculations taking into account both, the peculiar energy spectrum and collision broadening effects within the self-consistent Born approximation have been carried out and are presented in this issue [12]. The magnitude of the band-conductivity depends on $V_{0}$ and $\Gamma$ in a complicated manner and may dominate the scattering rate contribution or not. It is shown that, as in experiment, both situations are possible.

The suppression of the band-conductivity contribution as a function of increasing magnetic field is displayed in fig. 3. All data have been taken at $4.2 \mathrm{~K}$. For $V_{\mathrm{g}}=+150$ and $+200 \mathrm{mV}$ clear band-conductivity oscillations emerge around $0.15 \mathrm{~T}$ but are suppressed if the magnetic field is increased above $0.25 \mathrm{~T}$. In the view of the picture given above this means that the subband splitting of LL's with high quantum numbers ( $n$ $=27$ for $V_{\mathrm{g}}=+100 \mathrm{mV}$ ) is not sufficiently resolved with respect to collision broadening. This is consistent with the $(n X)^{-1 / 4}$ decay of the modulation bandwidth. For $V_{\mathrm{g}}=+300 \mathrm{mV}$ where the

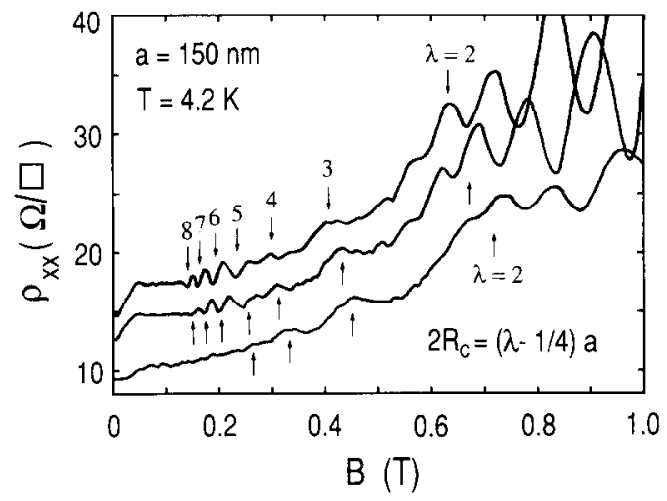

Fig. 3. $\rho_{x x}$ versus $B$ for $V_{\mathrm{g}}=+150$, +200 , and $+300 \mathrm{mV}$ (from top to bottom). The mobility increases from $1.7 \times 10^{6}$ $\mathrm{cm}^{2} / \mathrm{Vs}$ to $2 \times 10^{6} \mathrm{~cm}^{2} / \mathrm{Vs}$. Switching from "band-conductivity controlled" (minima at flat bands) oscillations to "scattering rate controlled" (maxima at flat bands) oscillations occurs at $\sim 0.25 \mathrm{~T}$ for both upper traces. For $V_{\mathrm{g}}=+300 \mathrm{mV}$, bandconductivity is completely suppressed. $\mathrm{SdH}$-oscillations emerge around $0.6 \mathrm{~T}$.

mobility is increased to a value of $\sim 2 \times 10^{6}$ $\mathrm{cm}^{2} / \mathrm{Vs}$ band-conductivity oscillations are effectively suppressed.

Only an indirect manifestation of the internal LL-structure could be given so far. Around 0.54 $\mathrm{T}\left(\Phi / \Phi_{0}=3\right)$ in fig. 2c a LL should split into three subbands. Although the temperature is low enough ( $k T \sim 5 \mu \mathrm{eV})$ to "scan" the Landau bands (whose width might be of the order of $100 \mu \mathrm{eV}$ ) no structure is resolved within those Landau levels. However, small inhomogeneities in the carrier density, e.g., can cause "averaging" over one LL. Only $\sim 7 \%$ of the carriers are within a $\mathrm{LL}$ at $0.54 \mathrm{~T}$ in fig. 2 . Fluctuations on the order of $1 \%$ can therefore easily prevent the direct observation of the internal LL-structure in macroscopic samples while the suppression of the band-conductivity indicates their existence on a mesoscopic length scale. By going towards smaller periods, and shifting therefore $\Phi / \Phi_{0}=1$ to higher magnetic fields the internal structure of the LL's should become directly observable.

We thank R.R. Gerhardts, D. Pfannkuche, and $\mathrm{U}$. Wulf for many fruitful discussions and $\mathrm{S}$. Koch for supporting the mK-measurements. 


\section{References}

[1] D. Weiss, K. von Klitzing, K. Ploog and G. Weimann. Europhys. Lett. 8 (1989) 179; also in: High Magnetic Fields in Semiconductor Physics II. Vol. 87 of Springer Series in Solid State Sciences, Ed. G. Landwehr (Springer. Berlin, 1989) p. 357.

[2] R.R. Gerhardts, D. Weiss and K. von Klitzing, Phys. Rev. Lett. 62 (1989) 1173.

[3] (a) C. Zhang and R.R. Gerhardts, Phys. Rev. B 41 (1990) 12850.

(b) P. Vasilopoulos and F.M. Peeters, Phys. Rev. Lett. 63 (1989) 2120 .

[4] T. Ando and Y. Uemura, J. Phys. Soc. Jpn. 36 (1974) 959.

[5] R.W. Winkler, J.P. Kotthaus and K. Ploog, Phys. Rev. Lett. 62 (1989) 1177.

[6] C.W.J. Beenakker, Phys. Rev. Lett. 62 (1989) 2020

[7] A. Rauh, Phys. Status Solidi B 65 (1975) K9.
[8] D.R. Hotstadter, Phys. Rev. B 14 (1976) 2239, and references therein.

[9] R.R. Gerhardts, D. Weiss and U. Wulf, Phys. Rev. B 4.3 (1991) 5192;

R.R. Gerhardts, D. Pfannkuche, D. Weiss and U. Wult, in: High Magnetic Fields in Semiconductor Physics III, Springer Series in Solid State Sciences, Ed. G. Landwehr (Springer, Berlin, 1991), in press.

[10] D. Wciss, K. von Klitzing and K. Ploog, Surf. Sci. 224 (1990) 88 .

[11] A number of papers emphasize this semi-classical aspect: See, e.g., P. Streda and A.H. MacDonald, Phys. Rev. B 41 (1990) 11892 .

P.H. Beton, M.W. Dellow, P.C. Main, E.S. Alves, L. Eaves, S.P. Beaumont and C.D.W. Wilkinson. Phys. Rev. B 43 (1991) 9980, and references therein.

[12] R.R. Gerhardt and D. Pfannkuche. Surf. Sci. 26.3 (1992) 324. 